\title{
Laser Material Processing in the Micro and Nanometer Domains: Past, Present and Possibly the Future
}

\author{
Henry Helvajian \\ Physical Sciences Laboratory, The Aerospace Corporation, MS:M2/241, P.O. Box 92957, \\ Los Angeles, CA 90009, USA \\ E-mail: Henry.Helvajian@aero.org
}

\begin{abstract}
An overview of the vast array of the laser material processing techniques is presented as they apply to fabricating structures in the micro and nanometer scale length domains. The overview covers developments from the past 20 years, and encompasses demonstrated processes where the use of the inherent properties of lasers has led to increased fidelity in the processing of materials. These demonstrated processes often use inventive approaches that rely on derivative aspects of established primary principals that govern laser/material interaction phenomena. By using these nuances in concert with the unique properties of lasers, a high level of finesse has been demonstrated in materials processing. The overview highlights by the use of selective examples those processes or techniques that appear to show scalability to the industrial environment.

DOI: 10.2961/j1mn.2009.01.0001
\end{abstract}

Keywords: laser processing, overview, micro/nanofabrication,

\subsection{Introduction}

Lasers have been used in materials processing for over 50 years, though in the early days, work was more focused on mitigating laser damage in materials as opposed to the processing of materials. However, the application of lasers to controllably alter materials was recognized early and as an industry, laser processing applications now lead world wide laser sales. The calendar 2005 totals for laser process tooling is near $\$ 6.0 \mathrm{~B} \mathrm{USD}^{1}$. Consequently, laser materials processing can no longer be considered a niche industry as there appears to be an increasing demand to apply laser processing solutions to manufacturing for specific value added segments. Commensurate with these industry trends is the fact that the number of conferences devoted to laser material processing continues to grow. This is a normative signal that industry is currently more amenable to applying laser based solutions into the manufacturing process. There are many factors that have contributed to this change in view over the course of the past 2 decades, the primary one being the advance of laser technology with a commensurate increase in system reliability.

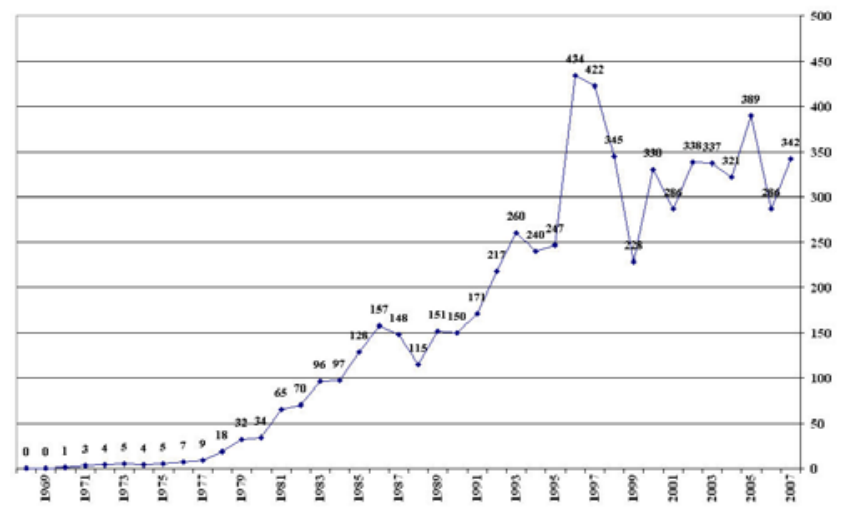

Figure 1: Publications per year laser materials processing.
Figure 1 shows a graph that depicts the number of world wide publications per year that mention laser material processing in the title or the abstract. The data spans nearly 40 years. The results represent a lower value to the total number of publications because there could be publications that discuss laser material processing research or development but fail to mention it in the title or abstract. The data is from an assembled series of databases that includes not only journal articles but also government sponsored research articles from the USA, Japan (e.g. MITI), Germany (e.g. BMFT), France (e.g. CNRS), Canada (e.g. NRC), UK (e.g. Department of Industry) and others. This data supports the conclusion that laser material processing is an established field that could support nearly 400 publications per year.

In this paper we explore a small segment of the overall laser material processing industry, namely the use and application of lasers in micro and nanofabrication. By their very nature of being a directed energy source, lasers have been the processing tool of choice when site-specific processing was desired. However, beyond just being able to deliver energy to a spot, much more sophisticated techniques and processes have been demonstrated for micro and nanofabrication. Thus, allowing the laser to be re-classified as truly a multifunctional tool. The goal of this paper is to present, by example, a select number of processes and techniques that enable the controlled fabrication/processing in the micro/nano dimensions which can also be conceivably scalable to the industrial environment. The review does not place emphasis on a particular type of laser or a particular application, as for example, the two recent and excellent reviews on the application of femtosecond lasers to bulk modification of 
transparent materials ${ }^{2}{ }^{3}$. This review casts a broader net over the possible techniques that have been developed in order to gain advantage in fabricating/processing in the micro/nano scale domain. The review employs the commonly known laser processing parameters (i.e. wavelength, power, dose, etc.) as an inventory of possible control knobs and describes the techniques in terms of these control parameters.

All reviews have the short coming of not capturing all the important research in one document. This review fares no better and in comparison is possibly worse given the strict limitation of space.

\subsection{Laser Processing}

The basic intent of laser material processing is to use the energy/force of the laser electromagnetic radiation to alter a material property in a desirable and controllable manner. This action entails the delivery of the energy to the material at the right time and place to ensue the desired light/matter interaction. The fundamental initial interaction can be via an absorber present in/on the material (e.g. dopant or chromophore) or via an induced or transient excitation because of the high intensities achievable with lasers. Regardless, the initial light/matter interaction is always via an electronic excitation that quickly decays by electronic-phonon coupling to result in local heating. Therefore, the physics/chemistry of laser material processing is nearly always a coupled phenomenon of electronic and thermal events. To produce a predominantly electronic process requires the exercising of experimental controls that have high fidelity. Ironically, this is easier to do in the nanometer scale, less so in the micrometer scale and even more difficult in larger dimension scales. A common approach is to minimize thermally mediated processes by utilizing the time dimension to advantage (i.e. short pulse or femtosecond laser processing) or by utilizing a specific photochemical/physical process (e.g. bond scission). The use of a short pulse to minimize thermal excitation has been successfully applied in the scission of living biological samples ${ }^{4}{ }^{5}$. However, it is also possible to do high precision materials processing via a thermally mediated action. This is feasible because thermally mediated processes can be calibrated with more ease over larger dimensional areas (e.g. controlled stress-induced bending). This technique has been successfully used to make sub-micron precision adjustments with multiple degrees of freedom in a microoptical system designed for digital audio/video recording systems ${ }^{6}$. The laser induced stress-bending technique enables precise tolerances to be achieved where accessibility by humans or other tooling is not feasible.

Over the years, laser material processing technology has realized improvements by using to advantage the properties of the laser light and beam delivery system. These properties include,

- Wavelength

- $\quad$ Energy, power fluence, irradiance
- $\quad$ Dose (e.g. number of applied laser shots)

- Processing beam character and spatial, temporal properties

- Laser pulse “train”

- Pattern generation approach

\subsection{Laser Wavelength}

In early applications, the established laser material processing approach was to choose the closest available laser wavelength that achieves the intended process with most effectiveness. As there were only a handful of fixed laser wavelengths that could be derived from reliable lasers, the wavelength choices were few. With the increase in the number of available laser wavelengths, contemporary research is now directed at exploring the advantages of micromachining with multiple laser wavelengths in a pump-probe configuration. These results have demonstrated that processing with multiple laser wavelengths results in better micromachining. For example, in the late 1990's experiments conducted in Japan demonstrated the power of multi-wavelength laser processing on wide band gap materials (e.g. fused silica ${ }^{7}$ and $\mathrm{SiC}, \mathrm{GaN}^{8}$ ). The experiments used a combination of UV and VUV laser sources that were co-aligned and where the higher power UV laser photon energy was below the material bandgap energy. In the particular case of the fused silica studies, a portion of the $266 \mathrm{~nm}$ Nd-Yag laser was antiStokes Raman shifted to generate VUV light. Clearly the technique applies a nuance to the standard laser machining approach, but it is a nuance than enhances the process and it can be applied in the industrial environment because it only requires one laser. The conclusions reached were that the multi wavelength ablation approach yielded a better surface finish in the ablated material ${ }^{7}$. In a more recent systematic study on silicon using nanosecond and picosecond lasers, similar conclusions were reached in that multicolor ablation can provide a higher ablation yield and leave a better surface finish ${ }^{9}$. In the silicon work, using ns lasers at $1064 \mathrm{~nm}+532 \mathrm{~nm}$, a $160 \%$ enhancement in the ablation rate has been measured. These results can be understood from the perspective of the photophysical light/matter interaction, if the initial laser pulse (i.e. pump) dynamically alters the material absorption properties to enhance the absorption of the second laser wavelength then more energy can be controllably deposited in the material via two color processing approach. A potential extension of two color processing is chirp-pulse processing where there is a temporal distribution of a wavelength band. Chirp-pulse processing requires a large bandwidth that is commonly present in femtosecond lasers. A recent experiment in support of chirp-pulse processing is the fabrication of embedded waveguides of circular cross section in phosphate glass ${ }^{10}$. In this example, a positive chirp (i.e. low frequencies arrive first) generated the most circular waveguide.

\subsection{Laser Power}

A major processing control parameter is the on-target laser power (W), which can also be represented in terms of fluence $\left(\mathrm{J} / \mathrm{m}^{2}\right)$ or irradiance $\left(\mathrm{W} / \mathrm{m}^{2}\right)$ depending on the 
application. The established approach has been to maintain this parameter constant and within the processing window. However, recent efforts have shown that by the controllable variation of this parameter processing advantages could be derived in which different functional properties of the exposed base material can be realized by mere consequence of the irradiation conditions ${ }^{11}$. There are at least two viable approaches to providing high fidelity control of this laser parameter. One is the use of an external laser cavity electro optic modulator (e.g. Pockels or acousto optic device) for varying the laser amplitude or power of a single laser beam ${ }^{12}$. A second approach that could have more profound applications is by applying the techniques used for coherent laser beam combining ${ }^{13}$ but with the additional feature of modulating the individual laser beam powers. The power modulation would be done before beam combining.

A parameter connected with the laser power is the process dose which is commonly defined as the number of laser shots for pulsed lasers and the exposure duration length for CW lasers. For micro and nanofabrication, current approaches have found that better results, in terms of surface finish and morphology, are possible with lower laser fluences and large number of laser pulses (or for CW laser processing, short duration with repeated exposures). These conclusions were made clear in the late 1990's by work in Canada on the laser chemical assisted etching of $\mathrm{InP}^{14}$. The results show that lower laser fluence results in a much smoother processed surface. At the time, pulsed UV laser repetition rates were well below the $\mathrm{MHz}$ capabilities that are available today. Therefore, applications where a large number of low fluence pulses would be needed would have been impractical (i.e. not cost effective) from an industrial perspective. However, laser repetition rates have increased to $\mathrm{MHz}$ with tabletop systems at 10's of watts. More recent experiments further support the earlier conclusions by showing that the application of a large number of pulses produces better material. A particularly interesting example with large scale industrial applications is the laser synthesis of TiN functional coatings on pure titanium, by high repetition rate free electron laser processing ${ }^{15}$. On a smaller scale but of significant commercial value to the photonics industry is the isotropic $^{16}$ and birefringent ${ }^{17}$ refractive index changes observed in femtosecond laser processing in transparent materials. The index changes are commonly attributed to low fluence or threshold energy processes in contrast to observation of micro voids ${ }^{18} 19$ which is ascribed to an above threshold or higher energy process. More recently there is evidence that femtosecond lasers could affect the non linear index ${ }^{20}$. If the process could be controllable it would a very lucrative application in the development of integrated nonlinear photonics.

\subsection{Laser Beam}

The size and shape of the laser processing beam also serves as another processing parameter. Typical processing approaches are the use of single laser beams, Gaussian optics and processing in the far field with the limitation of diffraction effects. Contemporary techniques have displayed quite a bit of variety with regards to circumnavigating diffraction limitations. There has been ample research in the use of multiple laser beams and the use of interference effects to fabricate true three dimensional periodic structures ${ }^{21} 22$. The primary driving application being the desire to develop artificial crystals that have programmed photonic bandgaps ${ }^{23}$. There also have been elegant investigations on material processing in the optical near field (i.e. scanning near field optical microscopy; SNOM) ${ }^{24}$. Even though much fundamental photophysical/photochemical information can be derived from these material processing approaches, the practicality of scaling to larger processing areas still remains to be demonstrated. An interesting technique, though in the very preliminary development stages, but one that does have scalability is the conversion of the incident laser electromagnetic field to surface plasmon modes by which nanometer scale resolution processing can be realized. Plasmon modes have also been used to extend photolithography to sub wavelengths. Experiments have shown enhanced patterning resolution by exciting surface plasmons in a metallic mask that is in near contact with a substrate $^{25}$. Alternatively, another means to circumvent the limitations of diffraction in a mask lithography tool is the use of multiple exposures with Fourier masks. Two masks are used to collect and selectively image the low and high spatial frequencies separately ${ }^{26}$. Using this technique structures with sub $100 \mathrm{~nm}$ feature sizes have been fabricated using visible laser light. In all the above examples, the use of nonlinear optical excitation has been used wherever possible to advantage. Finally, although Gaussian beams and optics continue to dominate laser material processing in the micro/nanometer dimensions, there is an increasing use of Bessel beams to enable long depth of field exposures in laser direct write processing ${ }^{27}$.

\subsection{Laser Pulse Temporal Profile}

Until the advent of femtosecond lasers, the natural temporal profile of pulsed lasers were not altered except to remove temporal spikes from mode beating. Laser systems were developed with specific pulse temporal profiles in order to accommodate a specific application. The recent trend has been to develop widely tunable pulse width lasers for material processing; for example, there are laser systems that can be tuned from $4-20 \mathrm{~ns}^{28}$ or from $40 \mathrm{~ns}$ 300ns while maintaining constant energy ${ }^{29}$. Furthermore, with the advent of femtosecond lasers there is now the ability to alter the temporal profile on a more complex level through selective filtering of the bandwidth. Consequently, there has been renewed appreciation in developing complex temporal pulse shapes that enhance a specific process. Using a variant of a technique developed in the 1990s in the USA for femtosecond laser pulse shaping $^{30}$, experiments conducted in 2003 in Germany on dielectric materials (e.g. a- $\mathrm{SiO}_{2}, \mathrm{CaF}_{2}$ ) clearly show the value of shaping a pulse for micromachining applications ${ }^{31}$. The unintuitive result from that experiment is that a single uniform pulse profile may not necessarily yield the best machined structure. Another conclusion that can be drawn from that experiment which echoes prior work is the advantage of a train of laser pulses, albeit with short time 
delays (i.e. 500 fs), over a single shot. It has been known for some time that laser pulse trains result in better quality machining over single shot events. This concept was demonstrated in a systematic study in 1999 on fused silica where a burst of pulses (i.e. 430 mode locked pulses with 7.5 ns pulse to pulse separation) results in a higher quality ablation (i.e. less microcracking and shock induced effects) in comparison to a single high fluence laser pulse ${ }^{32}$. In a more recent investigation on steel, it was demonstrated that pairs of ns pulses also result in higher ablation rates over single pulses given the same energy density ${ }^{33}$. The data shows that there is a $100 \%$ increase in ablation rate for pairs of laser pulses where the first (i.e. pump) is the lower fluence pulse. Given these demonstrated advantages with multiple pulse processing, there has been a recent technology development to enable the selective amplitude modulation of each delivered laser pulse and to concurrently map the modulation profile with the toolpath pattern in a laser direct-write system ${ }^{34}$.

\subsection{Pattern Generation}

In laser material processing, patterns are generated either via lithography (masks and imaging) or via direct write patterning (use of a multi axis motion stage). A novel but practical extension to the mask lithography technique have been the use a dense array of mirrors ${ }^{35}$ or spatial light modulators ${ }^{36}$ both which are applied to realize a dynamic mask and the use of moving masks which move in synchronous with the laser pulse delivery (i.e. maskdragging $)^{37}$. The latter technique can produce a graded exposure structure while the former two techniques can in principle produce any structure. A Japanese group has successfully shown the application of a spatial light modulator to generate variable holograms for femtosecond laser processing ${ }^{36}$. A novel patterning scheme has also been demonstrated in which the properties of optical diffraction are used to generate the pattern ${ }^{38}$. Furthermore, there have been developments in the novel use of phase Fresnel lenses and holographic exposure techniques to make patterns of arbitrary features on a large scale ${ }^{22}$. In the specific area of direct-write patterning where the substrate or the laser beam is moved, there have been novel extensions to the basic idea. One primary development that appears to have wide applicability is patterning via laser induced forward transfer (LIFT) ${ }^{39}$. In this technique there is a transfer tape that holds the desired species to be transferred and the absorbed laser energy within the transfer tape forces the transfer of the material. This technique is reminiscent of pick-and-place machines used in the microelectronics industry but has been demonstrated in the transfer of metal ${ }^{40}$, electronic circuit elements ${ }^{41}$, liquid droplets ${ }^{42}$ and biological materials ${ }^{43}$. Other developed techniques that rely on laser direct write processing but appear to have practical applications are laser induced plasma assisted ablation (LIPAA) ${ }^{44}$, laser backside wet etching (LBWE) ${ }^{45}$ and three dimensional volumetric exposure patterning 164647 . In the case of LIPAA and LBWE, the substrate patterning process is assisted by the inclusion of an adjacent metallic surface (LIPAA) or a laser absorbing chemical reagent (LWWE). While in LIPAA the pattern is the consequence of direct ablation, in the latter application there is a laser induced chemical reaction. Although these techniques continue to be used and are being refined for industrial applications, a complementary set of techniques are being developed that show pattern resolution capability in the sub micron to nanometer dimensions. There has been some effort to apply molecular self-assembly techniques to pattern surfaces using a nanoparticle contact mask that can be washed after exposure ${ }^{48}$. Although this approach has the capability to pattern 2D structures with resolution near 100 $\mathrm{nm}$, it cannot potentially achieve resolution down to the molecular level. The potential for near molecular level patterning was revealed by the recent demonstration from Denmark ${ }^{49}$. That group has shown that UV light can be used to immobilize a large variety of protein molecules on thiolated quartz, gold or silicon surfaces. The immobilized proteins are sterically oriented and can be further functionalized to serve as molecular scaffolding for further growth by chemical reaction. Therefore, it is conceivable that by utilizing a combination of high resolution mask lithography, control of the exposure dose at or near the exposure threshold level, and control of the protein concentration, large scale patterning near the molecular limit could be achieved. A variant of this concept has recently been demonstrated on the micron size scale by a group in Greece using three photon polymerization of an organic/inorganic glass (OCMOCER) to form a scaffolding on which biologically active systems were patterned via photo biotin photolysis ${ }^{50}$.

\subsection{Conclusions}

Because of the limitations of space, this brief review fails to cover other techniques and processes in which the material/substrate is altered or the processing environment is controlled to enhance a particular laser material interaction. Those techniques cover a vast array of material manufacturing processes that directly alter the properties of materials to enhance a particular laser process. These include work on liquid and gas phase photochemical processes that have been developed, over decades, for various laser assisted processes in manufacturing (e.g. Laser chemical vapor deposition, laser assisted chemical etching). The recent development of hybrid organic/inorganic materials (e.g. ORMOCER and ORMOSIL) designed for laser processing serves as an example. Finally, there is the increasing development of laser induced precipitation or crystallization processes that transform an amorphous material and imbue it with a desired functionality. A review on these processing techniques would require a strong emphasis on photochemistry and material science which diverts attention from the desire in this paper to provide a global overview from the perspective of the laser as a processing tool.

In summary, what is clear is that the laser material processing community now supports a worldwide industry with sales measured in \$B USD. A number of textbooks, spanning over 20 years, now document the research and technology developments in precision micro and

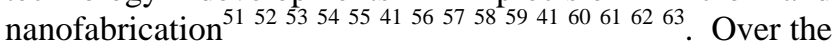


same time duration there have been dramatic strides in laser technology with smaller footprint, pulse stability and reliability being the current hallmarks and the controllable delivery of a prescribed photon flux being a capability on the horizon. In commensurate with this progress has been the development of a vast array of laser processing techniques that utilize the unique properties of the laser to evident advantage and thereby add value to the manufactured unit.

\section{Acknowledgements}

The author acknowledges The Aerospace Corporation's Independent Research and Development Program for providing funding to write this review. All trademarks, service marks, and trade names are the property of their respective owners.

\section{References}

1 A. Mayer, WILEY-VCH Verlag GmbH \& Co. KGaA, Weinheim (2006).

2 K. Itoh, W. Watanabe, S. Nolte, and C. B. Schaffer, MRS Bulletin 31, 620 (2006).

3 R. R. Gattass and E. Mazur, Nature Photonics 2, 219 (2008).

4 W. Watanabe, N. Arakawa, S. Matsunaga, T. Higashi, K. Fukui, K. Isobe, and K. Itoh, Optics Express 12 (18), 4205 (2004).

5 A. Vogel, J. Noack, G. Huttman, and G. Paltauf, Appl. Phys. B 81, 1015 (2005).

$6 \quad$ W. Hoving, Proc. SPIE 3097, 284 (1997).

7 K. Sugioka, S. Wada, Y. Ohnuma, A. Nakamura, H. Tashiro, and K. Toyoda, Applied Surface Science 9698, 347 (1996).

8 J. Zhang, K. Sugioka, S. Wada, H. Tashiro, K. Toyoda, and K. Midorikawa, Applied Surface Science 127-129, 793-799 (1998).

9 S. Zoppel, J. Zehetner, and G. A. Reider, Applied Surface Science 253 7692-7695 (2007).

10 A. Ferrer, V. Diez-Blanco, A. Ruiz, J. Siegel, and J. Solis, Applied Surface Science 254 (4), 1121-1125 (2007).

11 Frank E. Livingston and H. Helvajian, Journal of Photochemistry and Photobiology A: Chemistry 182, 310-318 ( 2006).

12 F.E. Livingston, L.F. Steffeney, and H. Helvajian, Applied Surface Science 253, 8015-8021 (2007).

13 Bing Lei and Y. Feng, Optics Communications 281, 739-743 (2008).

14 C. E. Moffitt, J. M. Wrobel, D. M. Wieliczka, J. J. Dubowski, and J. W. Fraser, Proc. SPIE 274, 121 (1998).

15 P. SCHAAF, M. SHINN, E. CARPENE, and J. KASPAR, Proceedings of the 6th International Symposium on Laser Precision Microfabrication, 1 (2005).

16 K. M. Davis, K. Miura, N. Sugimoto, and K. Hirao, Optical Letters 21 (21), 1729 (1996).

17 L. Sudrie, M. Franco, B. Prade, and A. Mysyrowicz, Optics Communications 171, 279 (1999).
18 E. N. Glezer and E. Mazur, Appl. Phys. Lett. 71 (7), 882 (1997).

19 C. B. Schaffer, A. O. Jamison, and E. Mazur, Appl. Phys. Lett. 84 (9), 1441 (2004).

20 A. Zoubir, M. Richardson, L. Canioni, A. Brocas, and L. Sarger, J. Opt. Soc. Am. B 22 (10), 2138 (2005).

21 J.D. Joannopoulos, P. R. Villeneuve, and S. Fan, Solid State Communications 102 (2-3), 165 (1997); K. K. Seet, T. Kondo, V. Mizeikis, V. Jarutis, S. Juodkazis, and H. Misawa, Proc. of SPIE 6161, 616103 (2005).

22 S. Hasegawa, Y. Hayasaki, and N. Nishida, OPTICS LETTERS 31 (11), 1705 (2006).

23 E. Yablonovitch, Phys. Rev. Lett. 58 (20), 2059-2062 (1987); J. L. Ladison, J. J. Price, J. D. Helfinstine, and W. R. Rosch, presented at the Optical Microlithography XVIII, San Jose, CA, USA, 2004 (unpublished).

24 D. Bäuerle, G. Wysocki, L. Landström, J. Klimstein, K. Piglmayer, and J. Heitz, Proceedings of SPIE, 8 (2003).

25 D. B. Shao and S. C. Chen, APPLIED PHYSICS LETTERS 86, 253107 (2005).

26 S. R. J. Brueck, S. H. Zaidi, X. Chen, and Z. Zhang, Microelectronic Engineering 41-42, 145 (1998).

27 J. Amak, K. Yoshimura, D. Sawaki, and T. Shimoda, Proc. of SPIE, 497 (2005).

28 R. PATEL, M. L. STOCK, Z. SARTANIA, J. BOVATSEK, A. ARAI, and H. ENDERT, Proceedings of SPIE 4830, 352 (2003).

29 W. Wiechmann, L. Eyres, J. Morehead, J. Gregg, D. Richard, W. Grossman, and Proc. of 4th International Conference LAMP, 1 (2006).

30 A. M. Weiner, D. E. Leaird, J. S. Patel, and J. R. Wullert, Optical Letters 15 (6), 326 (1990).

31 R. Stoian, M. Boyle, A. Thoss, A. Rosenfeld, G. Korn, and I. V. Hertel, Proceedings of SPIE 4830, 435 (2003).

32 P. R. Herman, A. Oettl, K. P. Chen, and R. S. Marjoribanks, Proc of SPIE 3616, 148 (1999).

33 P.A. Pivovarov, S.M. Klimentov, V.I. Konov, and F. Dausinger, Proc. of SPIE 6606 66060Y (2007).

34 F. Livingston and H. Helvajian, Proceedings of the 6th International Symposium on Laser Precision Microfabrication (LPM2005) (2005).

35 W. G. Oldham and Y. Shroff, Microelectronic Engineering 73-74, 42 (2004).

36 Y. Hayasaki, T. Sugimoto, A. Takita, and N. Nishida, Applied Physics Letters 87 (3), 031101 (2005).

37 A. S. Holmes, M. E. Heaton, G. Hong, K. R. Pullen, and P. T. Rumsby, Proc. of the SPIE 5063, 152 (2003).

38 O. Van Overschelde, G. Guisbiers, and M. Wautelet, Applied Surface Science 253, 7890-7894 (2007).

39 Z. Tóth, T. Szörényi, and A. L. Tóth, Applied Surface Science 69 (1-4), 317 (1993).

40 Z. Kántor, Z. Tóth, and T. Szörényi, Applied Surface Science 86 (1-4), 196 (1995).

41 Direct-Write Technologies for Rapid Prototyping Applications Sensors, Electronics and Integrated Power Sources, edited by A. Pique and D. B. Chrisey (Academic Press, San Diego, CA, 2002). 
M. DUOCASTELLA, P. SERRA, J. M. FERNÁNDEZ-PRADAS, M. COLINA, and J. L. MORENZA, Proceedings of the 4th International Congress LAMP (2006) (2006).

C.Z. Dinu, V. Dinca, J. Howard, and D. B. Chrisey, Applied Surface Science 253, 8119-8124 (2007).

44 Y. Hanada, K. Sugioka, I. Miyamoto, and K. Midorikawa, Proc. of SPIE 5713, 445 (2005).

45 Y. Kawaguchi, H. Niino, T. Sato, A. Narazaki, and R. Kurosaki, Journal of Physics 59, 380-383 (2007).

46 E. N. Glezer, M. Milosavljevic, L. Huang, R. J. Finlay, T.-H. Her, J. P. Callan, and E. Mazur, Optics Lett. 2023 (1996)

47 W. W. Hansen, S. W. Janson, and H. Helvajian, Proceedings of SPIE 2991, 104 (1997).

48 F. Burmeister, W. Badowsky, T. Braun, S. Wieprich, J. Boneberg, and P. Leiderer, Applied Surface Science. 144-145, 461-466 (1999). M. Duroux, L. Duroux, M.T. Neves-Petersen, E. Skovsen, and S. B. Petersen, Applied Surface Science 253, 8125-8129 (2007).

50 M. Farsari, G. Filippidis, T. S. Drakakis, K. Sambani, S. Georgiou, G. Papadakis, E. Gizeli, and C. Fotakis, Applied Surface Science 253, 8115-8118 (2007).

51 D. Bäuerle, Laser Processing and Chemistry. (Springer, , Heidelberg, 1986).

52 Interfaces Under Laser Irradiation, edited by L. D. Laude, D. Bäuerle, and M. Wautelet (Martinus Nijhoff, Boston, 1987), Vol. 134
53 Laser Beam Interactions with Materials, edited by $\mathrm{M}$. von Allmen and A. Blatter (Springer, Heidelberg, 1987).

54 Laser Microfabrication: Thin Film Processes and Lithography, edited by D. J. Ehrlich and J. Y. Tsao (Academic Press, London, 1989).

55 W. M. Steen, Laser Material Processing. (Springer, London, 1991).

56 Laser Ablation: Principles and Applications, edited by J. C. Miller (Springer-Verlag, Heidelberg, 1994).

57 Laser Materials Processing, edited by L. Migliore (Marcel Dekker, New York, 1996).

58 H. G. Rubahn, Laser Applications in Surface Science and Technology. (Wiley-VCH, New York, 1999).

59 LIA Handbook of Laser Materials Processing, edited by J. F. Ready and D. F. Farson (Magnolia Publications, Orlando, FL, 2001).

60 N. I. a. A. M. Stoneham, Materials Modification by Electronic Excitation. (Cambridge Unversity Press, Cambridge, UK, 2001).

$613 D$ Laser Microfabrication: Principles and Applications, edited by H. Misawa and S. Juodkazis (Wiley-VCH, Weinheim, 2006).

62 Photon-based Nanoscience and Nanobiotechnology NATO ASI, Vol 239,, edited by J. J. Dubowski and S. Tanev (Springer, Heidelberg, 2006).

63 N.B. Dahotre and S.P. Harimkar, Laser Fabrication and Machining of Materials. (Springer, Heidelberg, 2008).

(Received: July 3, 2008, Accepted: February 3, 2009) 